Website: http://revistas.lamolina.edu.pe/index.php/acu/index

(C) Universidad Nacional Agraria La Molina, Lima - Perú

\title{
Determinación de la estabilidad de una pequeña embarcación pesquera de madera de diseño predominante en la zona norte del litoral peruano
}

\author{
Stability determination of small wooden fishing vessel of predominant design in north zone of pe- \\ ruvian littoral
}

\author{
Oscar Malpica Moreno ${ }^{1 *}$; José Bardales Azañero² \\ * Autor de correspondencia
}

\section{Resumen}

El objetivo del presente estudio es conocer la estabilidad y dinámica de una pequeña embarcación pesqueras de madera de 10,48 m de eslora, cuyo modelo es predominante en la zona norte del litoral peruano. Esta embarcación fue construida en la localidad de Constante, Distrito de Sechura, Región Piura. Las pruebas de inclinación se realizaron en la Caleta La bocana - Parachique. Para el cálculo de los brazos de adrizamiento GZ se utilizó el Método de Barnes que introduce en sus cálculos la corrección de capas y la integración radial, considerando siete condiciones de carga. Los resultados obtenidos de las curvas de estabilidad, corregidas de superficies libres, fueron evaluados considerando, principalmente, los requerimientos de estabilidad del "Código de Estabilidad sin averías" propuesto por la OMI, que es de aplicación para las embarcaciones pesqueras con esloras mayores a $12 \mathrm{~m}$. Los resultados también fueron evaluados frente a los requerimientos de otros Criterios de Estabilidad, que de acuerdo al tamaño de la embarcación, son exigidos en otros países. Los resultados finales obtenidos fueron muy satisfactorios, porque esta embarcación, a pesar de su tamaño, cumple con la mayoría de las exigencias consideradas.

Palabras clave: Embarcación pesquera; Estabilidad; Criterios de estabilidad; Embarcación artesanal; Embarcación de madera.

\begin{abstract}
Abstrac
The objective of this study was to know the static and dynamic stability of small wooden fishing vessels stability of 10,48 meters length overall, which is a predominant model in the littoral of Peruvian north zone. This vessel was constructed in Constante locality (Sechura District - Piura Region - Perú). The inclining experiments was realized in La Bocana cove (Parachique locality - Sechura District). For the GZ (righting lever) calculations was used the Barnes method with layers correction and radial integrations for siete load conditions. The results obtained from the free surface corrected stability curves, was evaluated considering the stability requirements proposed by the IMO in the International Code on Intact Stability, applied for fishing vessels having a length of 12 meters and over. The results, was also evaluated with the requirements of stability criteria applied in some other countries.

The final results obtained were greatly satisfactory because this fishing vessel, in spite of its size, fulfil with almost all of the stability criteria used.
\end{abstract}

Keyword: Fishing vessel; Stability; Stability Criteria; Artisanal boat; wooden boat.

\section{Introducción}

La pesca es una actividad que proporciona importantes fuentes de alimentos, nutrición, ingresos y medios de vida para cientos de millones de personas en todo el mundo. Se estimó que el número total de embarcaciones de pesca en el mundo en 2016, desde pequeñas embarcaciones sin cubierta y sin motor hasta grandes y complejos buques industriales, era aproximadamente de 4,6 millones. Al rededor del $86 \%$ de las embarcaciones motorizadas en el mundo se incluían en la categoría de menos de 12 metros de eslora con predominio en todas las regiones del mundo.
El número de embarcaciones a motor se estimó en 2,8 millones a nivel mundial en 2016, lo cual supone el 61 $\%$ de todas las embarcaciones de pesca, solo alrededor del $2 \%$ de todas las embarcaciones pesqueras con motor medían 24 metros o más de eslora. A escala mundial, la FAO estimó la existencia de unas 44600 embarcaciones de pesca con al menos 24 metros de eslora (FAO, 2018).

Ante una escalofriante cifra de muertes a bordo de embarcaciones pesqueras y en mayor porcentaje, a bordo de las embarcaciones con esloras menores de $12 \mathrm{~m}$, la FAO presentó un documento a la OMI proponiendo la búsqueda

\footnotetext{
${ }^{1}$ Departamento Académico de Manejo Pesquero y Medio Ambiente, Facultad de Pesquería, Universidad Nacional Agraria La Molina, Apartado postal 12-056 - La Molina, Lima, Perú. Email: osma@lamolina.edu.pe

${ }^{2}$ Departamento Académico de Manejo Pesquero y Medio Ambiente, Facultad de Pesquería, Universidad Nacional Agraria La Molina, Apartado postal 12-056 - La Molina, Lima, Perú. Email: josebardales@lamolina.edu.pe
} 
de normas de seguridad internacionales para este tamaño de embarcaciones; el MSC (Maritime Safety Commite), en su $79^{\circ}$ periodo de sesiones, acordó incluir en el programa de trabajo del Subcomité de Estabilidad y Líneas de Carga y Seguridad de Pesqueros (SLF 79) un nuevo punto de alta prioridad sobre la "Seguridad de los buques pesqueros pequeños". El objetivo era elaborar recomendaciones de seguridad para los buques con cubierta, de eslora inferior a $12 \mathrm{~m}$, y los buques sin cubierta, de cualquier eslora (FAO/ OIT/IMO, 2014).

El Perú es miembro integrante de la OMI desde 1968; como tal, asume la normativa principal del "Código de Estabilidad sin Averías 2008" que demuestra ser hasta ahora, una de las mejores herramientas para establecer márgenes de seguridad para las embarcaciones pesqueras.

El Perú es considerado uno de los países más ricos en recursos hidrobiológicos y la pesca es uno de los sectores más importantes, tanto por el aporte nutricional, como el impacto económico y social. Según la Ley General de Pesca (1992) y su Reglamento (Decreto Supremo Nº 0122001-PE), las embarcaciones pesqueras desarrollan dos actividades importantes, la pesca artesanal dedicada a la extracción de los recursos hidrobiológicos para consumo humano directo y la pesca industrial extrae los recursos para ser destinados principalmente al consumo humano indirecto (harina y aceite de pescado). Las embarcaciones artesanales son unidades con esloras menores de $15 \mathrm{~m}$ y una capacidad de bodega hasta $32,6 \mathrm{~m}^{3}$ (30 TM), autorizadas para pescar hasta las 5 millas náuticas.

De acuerdo al Primer Censo Nacional de la Pesca Artesanal en el Ámbito Marino 2012, en el litoral peruano existen 16231 embarcaciones pesqueras artesanales, de los cuales 5621 embarcaciones (32,9\%) se registran en la Región Piura, brindando trabajo a 14161 pescadores artesanales en dicha región (Magallanes, 2015).

A pesar del alto número de embarcaciones pesqueras en el litoral peruano, no existe estudio publicado referente a los accidentes ocurridos; tan solo existen los reportes de la Dirección de Capitanías y Guardacostas del Perú (DICAPI), por lo que sería de mucha utilidad realizar investigaciones más profundas para clarificar las causas y consecuencias de cada accidente (Mantari et al., 2011).

El presente trabajo se hace necesario, no solamente por el tamaño que representa la embarcación en estudio (10,48 m de eslora total), sino por la sencilla razón de que esta embarcación es parte de un grupo que se construyen artesanalmente en la zona norte del litoral peruano y que sus relaciones: Eslora/Manga y Eslora/Puntal, son menores y no guardan relación con las embarcaciones convencionales (Takagui, 1960).

El objetivo del presente estudio fue determinar la estabilidad estática y dinámica de la embarcación, sobre la base del análisis de los brazos adrizantes, bajo 7 condiciones de carga, analizados a la luz de los requerimientos de estabilidad exigidos por la OMI en el Código de Estabilidad sin Averías 2008 y los más importantes Criterios de Estabilidad existentes, como una contribución al conocimiento, la utilidad y difusión de la estabilidad de embarcaciones pesqueras menores de $12 \mathrm{~m}$ de eslora.

\section{Materiales y métodos}

\section{La embarcación pesquera}

La embarcación de estudio fue construida de madera, manteniendo las características propias de las denominadas "Embarcaciones Vikingas", que son un modelo predominante en la zona norte del litoral peruano, específicamente en las costas de las Regiones de Lambayeque y Piura. Esta embarcación se encuentra operando en la zona de Sechura, dedicada a la extracción de la anchoveta (Engraulis ringens) para consumo humano directo. Sus dimensiones principales son: $\operatorname{Lmax}=10,48 \mathrm{~m}$; $L p p=9,29 \mathrm{~m} ; \mathrm{M}=4,12 \mathrm{~m} ; \mathrm{Pd}=1,81 \mathrm{~m} ; \mathrm{Pc}=2,01 \mathrm{~m} ; \mathrm{TRB}$ $=19$ toneladas Moorsom; Capacidad de bodega $=16,83$ toneladas $\left(18,7 \mathrm{~m}^{3}\right)$.

\section{El plano de líneas de forma}

El plano de líneas de forma es la presentación convencional de la forma 3D del casco de una embarcación y constituye la herramienta principal para realizar los cálculos para las Curvas Hidrostáticas y las Curvas Cruzadas de Estabilidad. Inicialmente, la embarcación no contaba con este plano, por lo que fue necesario su elaboración sobre la base de mediciones realizadas en playa. En su elaboración se utilizó las herramientas de diseño del AutoCAD 2015. En la vista de perfil, se visualizan 10 secciones transversales, con 3 medios espacios en proa y 3 medios espacios en popa; y 6 niveles de flotación. En la vista de planta, se visualizan las proyecciones de las semimangas que conforman la mitad de las áreas de flotación (Figura 1).

\section{Las curvas hidrostáticas}

Son las curvas que reflejan el comportamiento geométrico de la carena recta de una embarcación para diferentes calados. Reciben el nombre de carenas rectas porque son calculadas en la condición de embarcación adrizada.

Las curvas son calculadas a partir de las semimangas obtenidas del Plano de Líneas de Forma (Figura 2) para cada uno de los niveles de flotación. Para el cálculo se utilizaron las Reglas de Integración Aproximadas de Newton-Cotes (1ra y 2da Regla de Simpson y la Regla de 5/8), siguiendo la metodología propuesta por (Barnaby, 1969 y Derret, 1999) y haciendo uso de las herramientas de cálculo del programa Excel 2013.

\section{La prueba de inclinación}

Prueba necesaria que permite conocer la altura metacéntrica (GM), y a partir de ella, la ubicación vertical del centro de gravedad $(\mathrm{KG})$ utilizando la relación:

$K G=K M-G M$

donde el KM se obtuvo de las Curvas Hidrostática (Figura $2)$. 


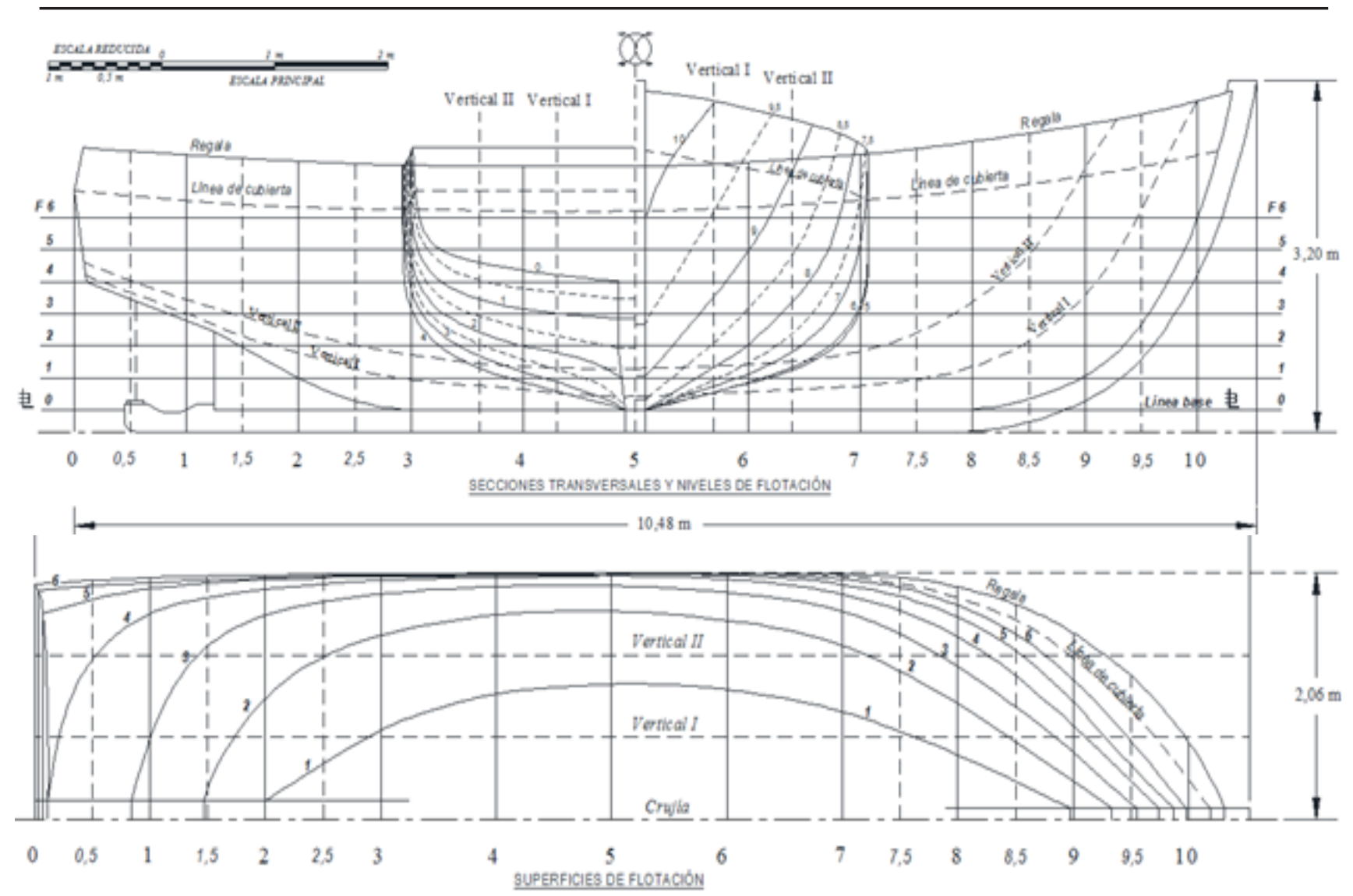

Figura 1. Plano de línea de forma. Embarcación Pesquera de madera de 10,48 m de eslora. Modelo predominante en la zona norte del Litoral Peruano

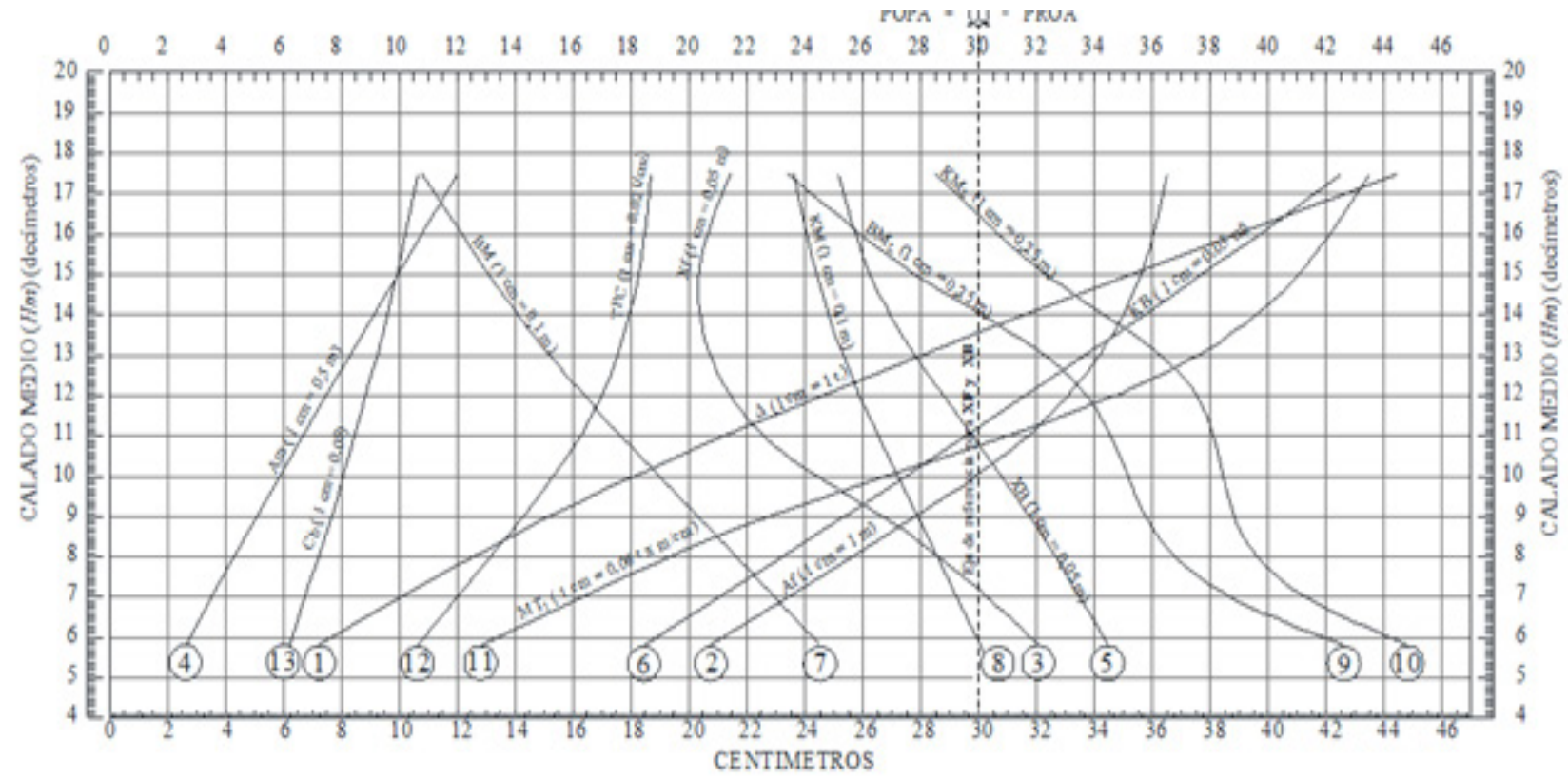

(1) Desplazamiento ( $\Delta)$ : Escala $1 \mathrm{~cm}=1 \mathrm{t}$

(2) Area de flotación (Af) : Escala $1 \mathrm{~cm}=1 \mathrm{~m}^{2}$

(3) Distancia de $\mathrm{F}$ al Cemtro de Eslora (XF): Escala $1 \mathrm{~cm}=0,05 \mathrm{~m}$

(4) Area de la Sección Maestra (Am): Escala $1 \mathrm{~cm}=0,5 \mathrm{~m}^{2}$

(5) Distancia de B al centro de eslora (XB): Escala $1 \mathrm{~cm}=0,05 \mathrm{~m}$

(6) Altura de B sobre LC (KB): Escala $1 \mathrm{~cm}=0,03 \mathrm{~m}$

(13) Coeficiente de block (Cb): Escala $1 \mathrm{~cm}=0,05$

Figura 2. Curva hidrostáticas. Embarcación Pesquera de madera de 10, 48 de eslora. Modelo predominante en la zona norte del Litoral peruano. $\mathrm{M}=$ Metacentro transversal; $\mathrm{M} 1=$ Metacentro longitudinal; $\mathrm{F}=$ centro de flotación; $\mathrm{B}=$ centro de carena; $\mathrm{K}=$ linea de la quilla 
Las pruebas tuvieron lugar en la Bahía de Sechura (Región Piura-Perú), donde se realizaron un total de 8 pruebas, utilizando para ello el método del péndulo (Díaz, 1972).

Los principales implementos utilizados para la prueba fueron:

- Un recipiente de $1,20 \mathrm{~m}$ x 0,65 m x 0,6 m;

- Un péndulo de aletas de $453 \mathrm{~g}$ de peso y $1,35 \mathrm{~m}$ de longitud de hilo trenzado;

- Una regla graduada en centímetros;

- Un peso de prueba de $180 \mathrm{~kg}$ repartido en 3 pesos parciales de $60 \mathrm{~kg}$, movidos a distancias transversales de $1,80 \mathrm{~m}$ a estribor y babor de crujía.

Participaron en la prueba 3 personas, dos ubicadas en cubierta cuya misión fue trasladar el peso, y uno en el piso de bodega para registrar el desvío del péndulo.

\section{Curvas cruzadas de estabilidad}

Se utilizó el Método de Barnes sobre la base de la aplicación de la Fórmula del momento de adrizamiento de Attwood, modificado para incluir la corrección de capas y el uso de la integración radial (Barnaby, 1969):

$$
\Delta G Z=\Delta\left\{\frac{\frac{2}{3} V_{i} y_{I} \times \cos \theta+\frac{2}{3} V_{e} y_{E} \times \cos \theta \pm v x}{V \pm v}-B G \times \operatorname{sen} \theta\right\}
$$

$\boldsymbol{G} \boldsymbol{Z}$ es el brazo adrizante (m); $\Delta$ es el desplazamiento (t); $\boldsymbol{V}_{i}$ y $\boldsymbol{V}_{e}$ son los volúmenes de las cuñas de inmersión y emersión respectivamente $\left(\mathrm{m}^{3}\right) ; \boldsymbol{v}$ es el volumen de la capa de corrección $\left(\mathrm{m}^{3}\right) ; \theta$ es la escora; $B G=$ distancia vertical entre el centro de carena y el centro de $\operatorname{gravedad}(\mathrm{m})$

En la toma de datos se utilizó un diseño de las secciones transversales completas ( Anexos 5) considerando en ella escoras de $0^{\circ}$ a $90^{\circ}$ a intervalos de $10^{\circ}(0,175 \mathrm{rad})$, en cada uno de los 6 niveles de flotación. Para los cálculos de áreas, volúmenes y momentos de inercia, se utilizaron la 1ra y 2da. Reglas de Simpson o la combinación de ambas (Anexos 1). Los brazos adrizantes supuestos $(G s Z s)$ fueron calculados asumiendo un centro de gravedad supuesto (Gs) a una altura de $1,7 \mathrm{~m}$ de la línea base $(K G s=1,7 \mathrm{~m})$.

\section{Condiciones de carga consideradas}

Se consideraron siete condiciones de carga: $\boldsymbol{C 1}=$ Salida del Puerto rumbo al caladero (con $100 \%$ de Combustible y agua dulce). $\boldsymbol{C} 2$ = Primera operación de cala (bodega vacía y $90 \%$ de combustible y agua dulce). $\boldsymbol{C} 3$ = Búsqueda del recurso (con $1 / 3$ de la bodega con pescado; $75 \%$ de combustible y agua dulce). $\boldsymbol{C} \boldsymbol{4}=$ Segunda operación de pesca (con $1 / 3$ de la bodega con pescado y $60 \%$ de combustible y agua dulce). $\boldsymbol{C 5}=$ Búsqueda del recurso (con 2/3 de la bodega con pescado y $45 \%$ de combustible y agua dulce). $\boldsymbol{C} \boldsymbol{6}=$ Tercera operación de pesca (con $2 / 3$ de la bodega con pescado y $30 \%$ de combustible y agua dulce). $\boldsymbol{C} 7=$ Retorno al Puerto (con la bodega llena de pescado y $20 \%$ de combustible y agua dulce).

Sobre la base del KG calculado mediante la prueba de inclinación, los factores necesarios $(\Delta, \mathrm{KG}, \mathrm{XG}, \mathrm{YG}, \mathrm{t}$, 486 etc.) para cada una de las condiciones de carga fueron obtenidos utilizando la metodología teórica de la adición, sustracción y traslación de pesos a bordo (Derret, 1999).

\section{Cálculo de los brazos adrizantes}

Los brazos adrizantes GZ para cada uno de los estados de carga fueron calculados a partir de los brazos adrizantes supuestos $(G s Z s)$ obtenidos de las Curvas Cruzadas de Estabilidad, ingresando con el desplazamiento, mediante la fórmula:

$G Z=G s Z s+-F F s x \operatorname{sen} \theta$

donde: GGs $x \operatorname{sen} \theta$ es el término de corrección, de signo positivo cuando el centro de gravedad verdadero $(\mathrm{G})$ está por debajo del centro de gravedad supuesto (Gs) y negativo en el caso contrario.

\section{Calculo de los brazos dinámicos}

Se define como estabilidad dinámica el trabajo utilizado para escorar la embarcación desde su posición de adrizado hasta una inclinación isocarena $\mathrm{q}_{1}$. En la curva de brazos adrizantes, la estabilidad dinámica para cualquier ángulo de escora se calcula multiplicando el bazo dinámico (área bajo la curva de brazos adrizantes) por el desplazamiento de la embarcación (Díaz. 1972):

$$
\Delta \times G Z_{\text {din }}=\Delta \times \int_{0}^{\theta} G Z d \theta
$$

Los brazos dinámicos para las 7 condiciones de carga fueron calculados de las curvas de brazos adrizantes, utilizando la Regla de 5/8 ( Anexos 3) (Derrett, 1999).

\section{Los criterios de estabilidad}

Para pequeñas embarcaciones pesqueras existen muy pocos criterios que permitan evaluar su estabilidad; aun cuando muchos de ellos demuestran una razonable adecuación, tienen una escasa base científica en su creación y continúan en uso básicamente debido a la falta de otros criterios más adecuados para este tamaño de embarcaciones (Womack, 2002).

Actualmente el Código de Estabilidad sin Averías, para todos los tipos de embarcaciones regidos por los Instrumentos de la OMI ofrece, en un solo documento, las disposiciones recomendadas en lo concerniente a la estabilidad, con la finalidad de garantizar la seguridad operacional de todas las embarcaciones y reducir al mínimo los riesgos para la propia embarcación, la tripulación y el medio ambiente (OMI, 2008).

\section{Criterios recomendados por la OMI}

La OMI recomienda a todos los países miembros, un conjunto de normas plasmadas en el Código de Estabilidad sin averías, para todos los tipos de embarcaciones, los criterios de estabilidad y otras medidas que garanticen su 
estabilidad y seguridad. Entre los criterios recomendados para las embarcaciones pesqueras menores de $24 \mathrm{~m}$ de eslora (aplicables a las embarcaciones de $12 \mathrm{~m}$ de eslora o más) se tiene: La altura metacéntrica inicial $\mathrm{GM}_{0}$ no deberá ser menor a $0,35 \mathrm{~m}$. El brazo adrizante (GZ) será como mínimo $0,20 \mathrm{~m}$ a una escora no menor de $30^{\circ}$. El máximo brazo adrizante $\left(G Z_{\max }\right)$ debe corresponder a una escora superior a $30^{\circ}$, pero nunca inferior a $25^{\circ}$. El área bajo la curva de brazos adrizantes, hasta una escora de $30^{\circ}$, no debe ser inferior a 0,055 m.rad. El área bajo la curva de brazos adrizantes, hasta una escora de $40^{\circ}$, no será inferior a 0,09 m.rad o hasta el ángulo de inundación $\mathrm{q}_{\mathrm{f}}$ si este fuera inferior a $40^{\circ}$. El área bajo la curva de brazos adrizantes entre las escoras de $30^{\circ}$ y $40^{\circ}$ o entre las escoras de $30^{\circ}$ y el ángulo de inundación $\mathrm{q}_{\mathrm{f}}$, si este fuera menor de $40^{\circ}$ no debe ser menor de $0,03 \mathrm{~m}$.rad. El Criterio de viento y balance intenso, conocido también como "Criterio Meteorológico", deberá ser aplicado a las embarcaciones pesqueras de eslora igual o superior a $24 \mathrm{~m}$ y que tengan una gran superficie expuesta al viento. Este criterio recomienda las disposiciones contenidas en la Parte A del Código de Estabilidad sin Averías, donde el área “ $\boldsymbol{b}$ ” deberá ser igual o superior al área " $a$ " calculados en las curvas de brazos adrizantes entre el brazos escorantes $\boldsymbol{l} \boldsymbol{w}_{2}$ y las verticales a la altura de los ángulos de escora $\theta_{1}$ y $\theta_{2}$ (Anexos 7).

El cálculo de $\boldsymbol{l} \boldsymbol{w}_{1 ;} \boldsymbol{l} \boldsymbol{w}_{2 ;} \mathbf{q}_{1}$ y $\mathbf{q}_{2}$ se realizaron utilizando las siguientes ecuaciones y las variables obtenidas de Anexos 4:

$$
\begin{aligned}
& l w_{1}=\frac{P \times A \times Z}{1000 \times g \times \Delta} \quad(\mathrm{m}) \\
& l w_{2}=1,5 \times l w_{1} \quad(\mathrm{~m}) \\
& \theta_{1}=109 \times k \times X_{1} \times X_{2} \times \sqrt{r \times s} \quad(\text { grados }) \\
& r=0,73+0,6 \times \frac{O G}{H m} \\
& T=\frac{2 \times C \times M}{\sqrt{G M}}(\mathrm{~s}) \\
& C=0,373+0,023 \times\left(\frac{M}{H m}\right)-0,043 \times\left(\frac{L f}{100}\right)
\end{aligned}
$$

donde: $P=$ presión del aire $\left(\mathrm{N} / \mathrm{m}^{2}\right)$ que para el presente caso equivale a $316 \mathrm{~N} / \mathrm{m}^{2} ; A=$ área lateral proyectada del casco y de la superestructura por encima de la línea de flotación en $\mathrm{m}^{2} ; Z=$ distancia vertical desde el centro del área " $A$ " hasta el centro del área lateral proyectada de la carena $(\mathrm{m}) ; \Delta=$ desplazamiento $(\mathrm{t}) ; g=$ aceleración de la gravedad $\left(\mathrm{m} / \mathrm{s}^{2}\right) ; X_{1}, X_{2}, k$ y $s$, obtenidos de Anexos 4; $O G=$ distancia entre el centro de gravedad y la línea de flotación (positivo si $\mathrm{G}$ está encima de la flotación y negativo en el caso contrario); $\mathrm{Hm}=$ calado medio (m); $\mathrm{T}$ = periodo de balance $(\mathrm{s}) ; L f=$ eslora de flotación $(\mathrm{m}) ; M=$ manga $(\mathrm{m}) ; A_{k}=$ área proyectada de la quilla en $\mathrm{m}^{2} ; G M=$ altura metacéntrica $(\mathrm{m})$.

\section{Criterio de Rahola}

Criterio ideado por el profesor finlandés Jaakko Juhani Rahola, publicado en su tesis doctoral en 1935, quien analizó la pérdida de numerosas embarcaciones por falta de estabilidad, sugiriendo un criterio basado en los brazos adrizantes (corregido de superficies libres) y el ángulo de inundación. A este criterio se le conoce como la "regla de los mínimos", porque establece los mínimos valores que debe tener los brazos adrizantes para ciertos valores dados de escora. Este criterio fue el precursor de todos los Criterios que ha publicado la OMI con posterioridad y recomienda (Womack, 2002):

- El máximo brazo adrizante $\left(\mathrm{GZ}_{\max }\right)$ debe estar situado entre $30^{\circ}$ y $40^{\circ}$ de escora.

- Los valores de GZ (corregido de superficies libres) deben estar por encima de la línea curva formada por los valores de GZ de $14 \mathrm{~cm}, 20 \mathrm{~cm}$ y $20 \mathrm{~cm}$ para escoras de $20^{\circ}, 30^{\circ}$ y $40^{\circ}$ respectivamente (Tabla 1 ).

- El brazo dinámico $\left(\mathrm{GZ}_{\mathrm{din}}\right)$ para $40^{\circ}$ de escora debe ser, como mínimo, de 0,08 m.rad.

Tabla 1. Valores mínimos de $G Z$ para ángulos de escora. Requerimiento de Rahola.

\begin{tabular}{|l|l|}
\hline $\mathrm{GZ}=14 \mathrm{~cm}$ & Escora $=20^{\circ}$ \\
$\mathrm{GZ}=20 \mathrm{~cm}$ & Escora $=30^{\circ}$ \\
$\mathrm{GZ}=20 \mathrm{~cm}$ & Escora $=40^{\circ}$ \\
\hline
\end{tabular}

\section{Criterio de Nickum}

Nickum, G. (1975), recomienda el siguiente criterio, aplicable para embarcaciones pesqueras en servicio:

- En la condición más crítica de trabajo, la altura metacéntrica (GM) debe ser igual o mayor que el $10 \%$ de la manga ó igual o mayor de 0,61 m y debe complementarse con las mediciones de GM calculado con la fórmula:

$G M=\left[\frac{k \times M}{T}\right]^{2}$

donde: $k=0,8 ; M=$ manga de trazado $(\mathrm{m}) ; T=$ periodo de oscilación completa (s).

- Debe cumplirse la relación: $\frac{f}{M}+\frac{A}{L p p \times M} \geq 0,15$ donde: $f=$ francobordo (m); $M=$ manga (m); $A$ = área de la obra muerta proyectada sobre el plano de crujía $\left(\mathrm{m}^{2}\right) ; L p p=$ eslora entre perpendiculares $(\mathrm{m})$

\section{Criterio Holandés}

Propone una única recomendación que debe ser aplicada a barcos que tengan un Tonelaje de Registro Bruto (TRB) menor de 500:

- En las peores condiciones de carga, la curva de brazos adrizantes debe cortar o ser tangente a una línea quebrada formada por los brazos adrizantes (GZ) de 0,40 m, 0,22 m y $0,27 \mathrm{~m}$ para las escoras de $0^{\circ}, 35^{\circ}$ y $60^{\circ}$ respectivamente (Díaz, 1972). 


\section{Criterio Japonés}

Criterio recomendado por la JFA (Agencia Pesquera del Japón), con referencia a la estabilidad y seguridad para pequeñas embarcaciones pesqueras (JFBA, 1964):

- En todas las condiciones de carga debe cumplirse que:

$G M \geq 0,04 \times M+\alpha \times \frac{M}{P d}-\beta$

donde: $\alpha=0,28$ (para embarcaciones de madera); $\beta=0,52$; $M=$ manga (m); $P d=$ puntal de diseño (m).

- Para el caso de embarcaciones cerqueras, el GM mínimo debe ser el mayor valor obtenido por una de las siguientes fórmulas:

$G M_{\text {min }}=M / 23+0,27 ; G M_{\text {min }}=L f / 120+0,27 ; G M_{\text {min }}=0,45 \mathrm{~m}$.

- En el caso de embarcaciones de madera, el francobordo mínimo $\left(f_{\min }\right)$ debe obedecer a la siguiente relación:

$f_{\text {min }} \geq p d / 15+0,20$

\section{Criterio de Takagui}

Takagui, A. (1960), luego de analizar el comportamiento de 96 embarcaciones pesqueras, recomienda como criterio para todos los tipos de barcos de pesca lo siguiente:

- La altura metacéntrica (GM) debe estar comprendido entre $45 \mathrm{~cm}$ y $60 \mathrm{~cm}$.

- En embarcaciones de madera, el periodo de balance (T) debe cumplir con la siguiente relación:

$T \leq 2 \times \frac{k t}{\sqrt{G M}}$

donde: $k t \mathrm{~m} * M ; 0,44 \leq m \leq 0,60$, siendo $M$ la manga en metros.

- La constante de estabilidad "C" obtenida por la fórmula (16), debe caer en la zona de seguridad (Anexos 12).

$$
C=[(G M / B G) *(2 * f / M)]
$$

donde $G M=$ altura metacéntrica $(\mathrm{m}) ; B G=$ distancia entre el centro de carena y el centro de $\operatorname{gravedad}(\mathrm{m}) ; f=$ francobordo (m); $M=$ manga $(\mathrm{m})$.

\section{Resultados y discusión}

Factores necesarios para el análisis de la estabilidad.

La prueba de inclinación y la prueba de balance arrojaron los resultados que se muestran en la Tabla 2, y a partir de estos resultados fueron calculados los factores requeridos por los Criterios de Estabilidad para cada una de las siete condiciones de carga (Tabla 3).

\section{Las curvas cruzadas de estabilidad}

Cada una de las curvas en la Figura 3, representan la variación de las escoras consideradas con respecto a los brazos adrizantes supuestos (GsZs) (Anexos 2) y el desplazamiento en cada uno de los 6 niveles de flotación considerados. Los valores que se muestran en la Tabla 2 de Anexos fueron calculados aplicando la metodología de Barnes y considerando un KGs de 1,7 m.

Tabla 2. Valores iniciales durante la prueba de inclinación, la altura metacéntrica hallada y la ubicación final del centro de gravedad $(\mathrm{KG})$

\begin{tabular}{lclc}
\hline \multicolumn{1}{c}{ Atributo } & Medida & \multicolumn{1}{c}{ Atributo } & Medida \\
\hline$\Delta$ (toneladas) & 23,846 & $K G(m)$ & 1,735 \\
$H m(m)$ & 1,061 & $G M(m)$ & 0,867 \\
$K M(m)$ & 2,602 & $T$ (segundos) & 3,998 \\
\hline
\end{tabular}

Tabla 3. Factores requeridos para la aplicación de los criterios de estabilidad para cada una de las siete condiciones de carga

\begin{tabular}{lcccccccc}
\hline \multirow{2}{*}{ Atributo } & \multicolumn{7}{c|}{ Condiciones de carga } \\
\cline { 2 - 9 }$\Delta$ & C1 & C2 & C3 & C4 & C5 & C6 & C7 \\
$\Delta$ & 26,42 & 24,76 & 31,85 & 30,14 & 37,23 & 35,55 & 42,59 \\
$H p r$ & $(m)$ & 1,13 & 1,11 & 1,22 & 1,26 & 1,3 & 1,34 & 1,42 \\
$H p p$ & $(m)$ & 1,38 & 1,3 & 1,6 & 1,46 & 1,81 & 1,68 & 1,95 \\
$H m(m)$ & 1,25 & 1,21 & 1,41 & 1,36 & 1,56 & 1,51 & 1,69 \\
$t \quad(\mathrm{~cm})$ & 25,4 & 19,9 & 39,2 & 23,18 & 51,5 & 35,9 & 63,6 \\
$K M$ & $(m)$ & 2,56 & 2,59 & 2,48 & 2,48 & 2,42 & 2,44 & 2,38 \\
$K B$ & $(m)$ & 0,98 & 0,95 & 1,08 & 1,08 & 1,16 & 1,14 & 1,25 \\
$K G$ & $(m)$ & 1,66 & 1,62 & 1,56 & 1,49 & 1,54 & 1,51 & 1,58 \\
$G M$ & $(m)$ & 0,90 & 0,97 & 0,92 & 0,99 & 0,88 & 0,93 & 0,80 \\
$B G$ & $(m)$ & 0,51 & 0,47 & 0,53 & 0,46 & 0,36 & 0,33 & 0,18 \\
$f$ & $(m)$ & 0,82 & 0,87 & 0,67 & 0,71 & 0,52 & 0,56 & 0,38 \\
$X F$ & $(m)$ & 0,45 & 0,43 & 0,48 & 0,48 & 0,48 & 0,48 & 0,44 \\
\hline
\end{tabular}

$\overline{\mathrm{C} 1}, \mathrm{C} 2, \ldots \mathrm{C} 7$ son las siglas que identifican cada una de las condiciones de carga descritas en el acápite 2,$5 ; \mathrm{Hpr}, \mathrm{Hpp}$ y $\mathrm{Hm}$, simbolizan los calados de proa, popa y el calado medio respectivamente; $\mathrm{t}=$ asiento; $G M=$ altura metacéntrica; $B G=$ distancia vertical entre el centro de gravedad y el centro de carena; $K G=$ distancia vertical del centro de gravedad respecto a la quilla; $f=$ francobordo. Con respecto a los símbolos $\Delta, K M$ y $K B$, (ver descripción en Figura 2).

\section{Las curvas de estabilidad}

De las Curvas Cruzadas de Estabilidad (Figura 3), entrando con el desplazamiento de cada condición de carga, se obtuvieron los correspondientes brazos adrizantes supuestos $(G s Z s)$, los que fueron corregidos utilizando la fórmula (3) y obteniéndose de esa manera las curva de brazos adrizantes. A partir de la curva de brazos adrizantes se obtuvieron las curvas de brazos dinámicos para cada una de las siete condiciones de carga (Figura 4).

\section{Criterios recomendados por la OMI}

El valor en negrita no cumple con el mínimo establecido por el criterio. En la Tabla 4 se observa que los requerimientos de GM y brazos dinámicos $\left(\int_{\theta_{1}}^{\theta_{2}} G Z d \boldsymbol{C}\right)$ son cumplidos satisfactoriamente; la exigencia de que el máximo brazo adrizante ocurra a escoras mayores a $30^{\circ}$ pero nunca a escoras menores de $25^{\circ}$ son cumplidas satisfactoriamente. 


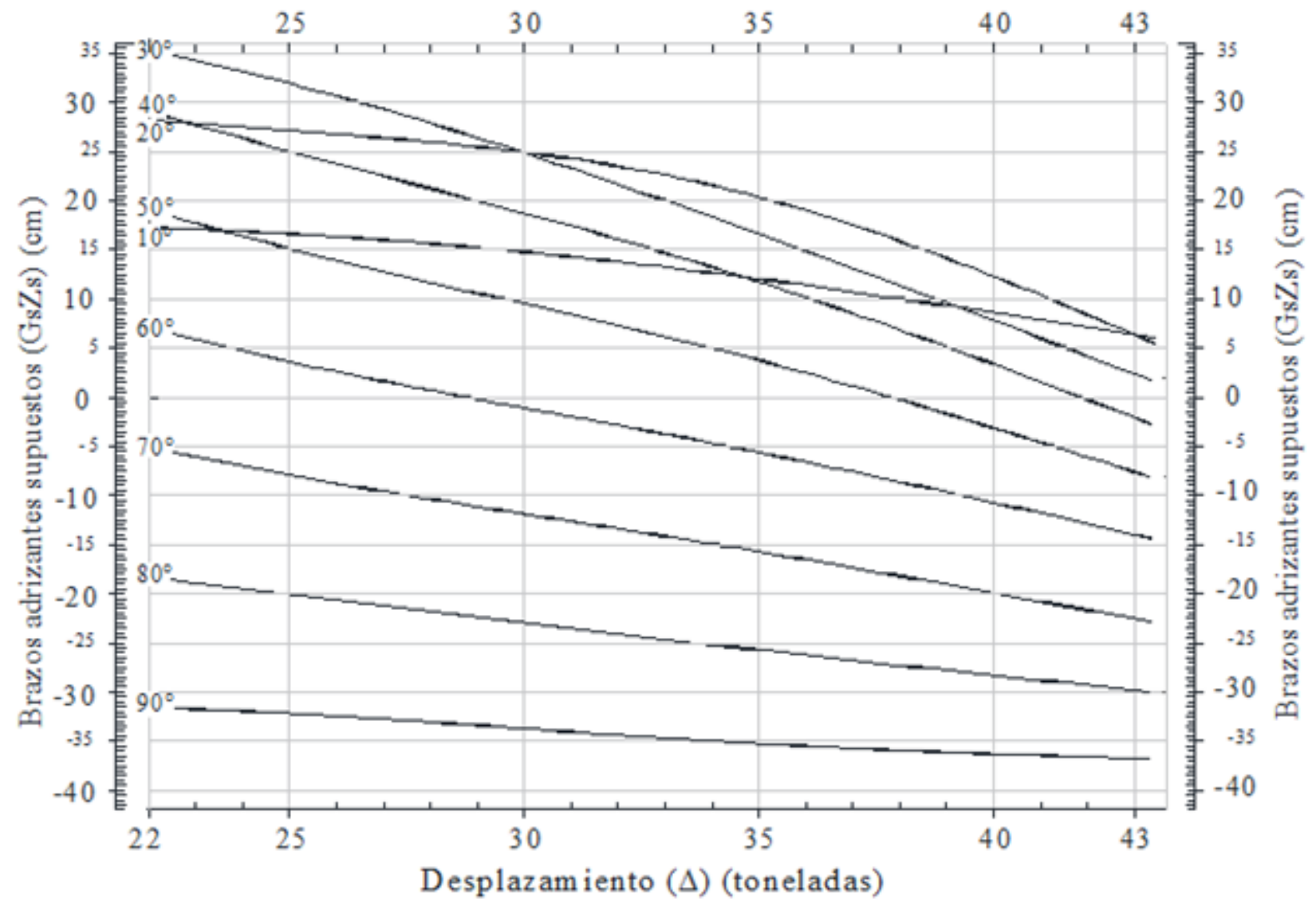

Figura 3. Curvas cruzadas de estabilidad $\mathrm{KGs}=1,7 \mathrm{~m}$

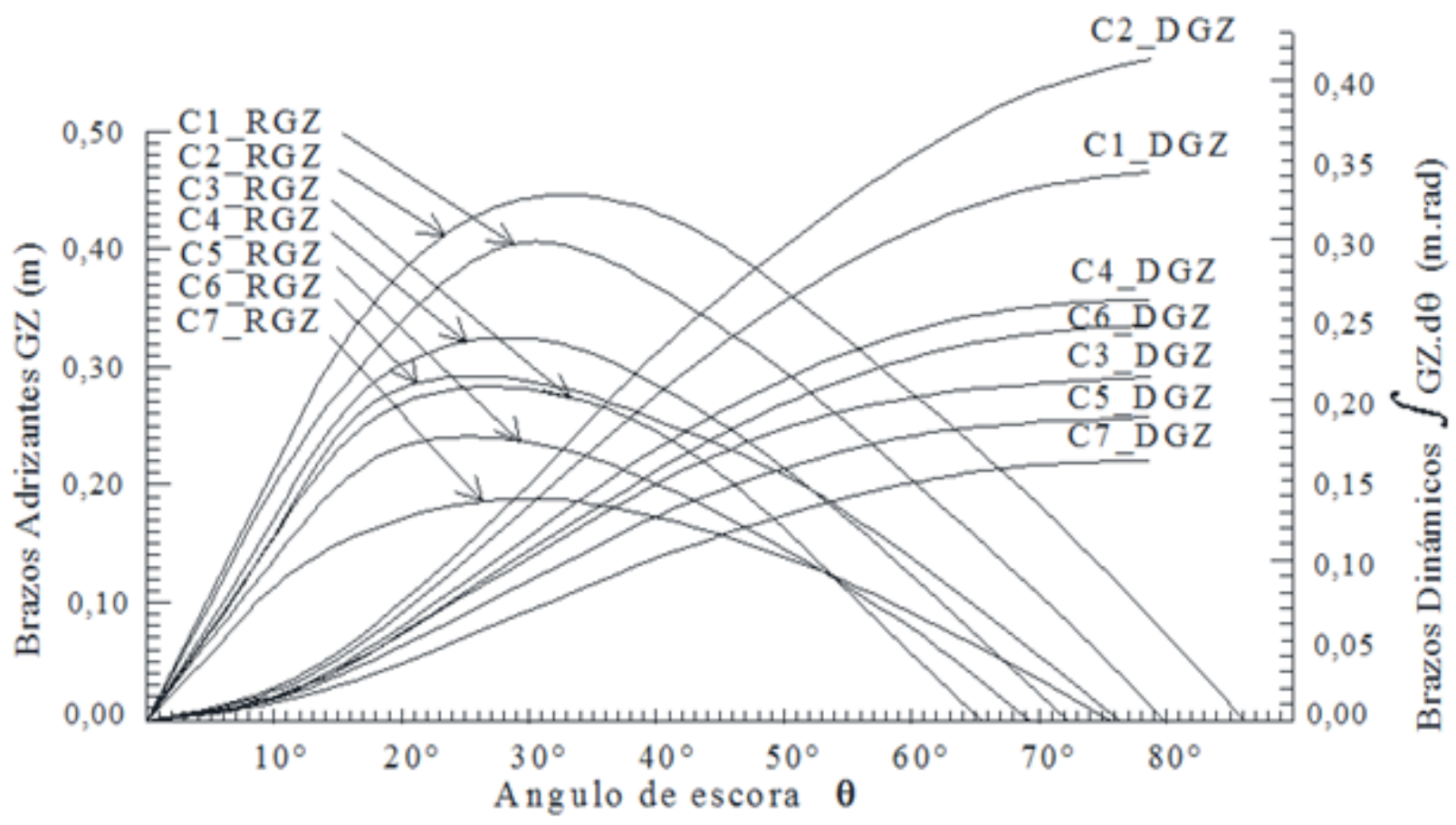

Figura 4. Curvas de brazos adriazantes y curvas de brazos dinámicos para las siete condiciones de carga. C1, C2, C7 simbolizan las siete condiciones de carga (definidos en el Acápite 2,5). C1_RGZ, C2_RGZ ... C7_RGZ simbolizan las curvas de brazos dinámicos 
Los brazos adrizantes obtenidos en cada una de las condiciones de carga para escoras mayores de $30^{\circ}$, superan los $0,20 \mathrm{~m}$ excepto en la condición de carga C7 que alcanza solamente $0,18 \mathrm{~m}$. La Tabla 5 resume las áreas "a" y "b" calculadas para cada una de las condiciones de carga, observándose que todas las áreas "b" son mayores que las áreas "a", cumpliendo satisfactoriamente con la exigencia.

\section{Criterio de Rahola}

Los números en negrita son los valores que no cumplen con los requerimientos del criterio. La magnitud de los brazos adrizantes requeridos por el Criterio de Rahola (Tabla 6), son menores a los exigidos, solamente en la condición de carga C7 (retorno al Puerto con $100 \%$ de captura en bodega y $10 \%$ de combustible y agua dulce).

Tabla 4. Resultados de los valores calculados para la embarcación referentes a los valores mínimos requeridos por la $O M I$ (Altura metacéntrica (GM), Brazos adrizantes (GZ) y los Brazos dinámicos

\begin{tabular}{|c|c|c|c|c|c|c|c|}
\hline \multirow{2}{*}{ Requerimientos del Criterio } & \multicolumn{7}{|c|}{ Condiciones de carga } \\
\hline & $\mathrm{C} 1$ & $\mathrm{C} 2$ & $\mathrm{C} 3$ & $\mathrm{C} 4$ & $\mathrm{C} 5$ & C6 & $\mathrm{C} 7$ \\
\hline$G M_{0} \geq 0,35 \mathrm{~m}$ & 0,90 & 0,97 & 0,92 & 0,99 & 0,88 & 0,93 & 0,80 \\
\hline$G Z \geq 0,20 \mathrm{~m}$ en $\theta \geq 30^{\circ}$ & 0,41 & 0,44 & 0,28 & 0,32 & 0,24 & 0,29 & 0,18 \\
\hline$G Z_{\max }$ en $\theta \geq 30$ y no $<25^{\circ}$ & $30,5^{\circ}$ & $32,7^{\circ}$ & $27,0^{\circ}$ & $28,5^{\circ}$ & $25,6^{\circ}$ & $25,5^{\circ}$ & $30,0^{\circ}$ \\
\hline $\int_{0^{\circ}}^{30^{\circ}} G Z d \theta \geq 0,055 \mathrm{mrad}$ & 13 & 15 & 0,10 & 011 & 0,09 & 0,11 & 0,07 \\
\hline $\int_{0^{40}}^{40^{\circ}} G Z d \theta \geq 0,09$ & 0,20 & 0,23 & 0,15 & 0,19 & 0,13 & 0,15 & 0,10 \\
\hline $\int_{30^{\circ}}^{40 \circ} G Z d \theta \geq 0,03 \mathrm{mrad}$ & 0,07 & 0,08 & 0,05 & 0,08 & 0,04 & 0,04 & 0,03 \\
\hline
\end{tabular}

Tabla 5. Resultados obtenidos de las áreas " $b$ " y " $a$ " (requerimiento del Criterio Meteorológico) para cada una de las condiciones de carga

\begin{tabular}{|c|c|c|c|c|c|c|c|}
\hline \multirow{2}{*}{$\begin{array}{c}\text { Requerimientos del } \\
\text { Criterio }\end{array}$} & \multicolumn{7}{|c|}{ Condiciones de carga } \\
\hline & $\mathrm{C} 1$ & $\mathrm{C} 2$ & $\mathrm{C} 3$ & $\mathrm{C} 4$ & $\mathrm{C} 5$ & C6 & $\mathrm{C} 7$ \\
\hline Area “b” (m.rad) & 0,07 & 0,08 & 0,05 & 0,06 & 0,04 & 0,05 & 0,03 \\
\hline Area “ $a$ ” (m.rad) & 0,04 & 0,05 & 0,03 & 0,04 & 0,03 & 0,03 & 0,02 \\
\hline
\end{tabular}

Tabla 6. Resultados de los brazos adrizantes y brazos dinámicos requeridos por el Criterio de Rahola

\begin{tabular}{lccccccc}
\hline \multirow{2}{*}{$\begin{array}{c}\text { Requerimientos del } \\
\text { Criterio }\end{array}$} & \multicolumn{7}{c}{ Condiciones de carga } \\
\cline { 2 - 8 } & $\mathrm{C} 1$ & $\mathrm{C} 2$ & $\mathrm{C} 3$ & $\mathrm{C} 4$ & $\mathrm{C} 5$ & $\mathrm{C} 6$ & $\mathrm{C} 7$ \\
\hline$G Z_{20^{\circ}} \geq 0,14 \mathrm{~m}$ & 0,34 & 0,38 & 0,27 & 0,30 & 0,22 & 0,28 & 0,17 \\
$G Z_{30^{\circ}} \geq 0,20 \mathrm{~m}$ & 0,41 & 0,44 & 0,28 & 0,32 & 0,24 & 0,29 & 0,18 \\
\hline
\end{tabular}

Tabla 7. Resultados de la altura metacéntrica de la embarcación comparado con los requerimientos del Criterio de Nickum

\begin{tabular}{|c|c|c|c|c|c|c|c|}
\hline \multirow{2}{*}{ Requerimientos del Criterio } & \multicolumn{7}{|c|}{ Condiciones de carga } \\
\hline & $\mathrm{C} 1$ & $\mathrm{C} 2$ & $\mathrm{C} 3$ & $\mathrm{C} 4$ & $\mathrm{C} 5$ & C6 & $\mathrm{C} 7$ \\
\hline$G M \geq 0,1 * M$ о $G M>0,61 \mathrm{~m}$ & 0,407 & 0,405 & 0,411 & 0,410 & 0,412 & 0,412 & 0,412 \\
\hline$G M=\left[\left(0,8^{*} M\right) / T\right]^{2}$ & 0,816 & 0,873 & 0,879 & 0,932 & 0,868 & 0,914 & 0,811 \\
\hline GM embarcación (m) & 0,902 & 0,974 & 0,922 & 0,986 & $\mathbf{0 , 8 7 8}$ & $\mathbf{0 , 9 3 0}$ & 0,803 \\
\hline$(\mathrm{f} / \mathrm{m})+\left(A / l f^{*} M\right) \geq 0,15$ & 0,658 & 0,698 & 0,567 & 0,592 & 0,494 & 0,512 & 0,429 \\
\hline
\end{tabular}

Igualmente el máximo brazo adrizante $\left(\mathrm{GZ}_{\max }\right)$ en las condiciones de carga C3, C4, C5 y C6 ocurren para escoras ores de $30^{\circ}$ (Anexo 9). Esta exigencia no es totalmente os requerimientos de brazos adrizantes en escoras de $20^{\circ}$ uede considerarse para calificar como una condición de

\section{Criterio de Nickum}

En la Tabla 7 se observa que los valores de la altura metacéntrica (GM) de la embarcación son mayores que los valores calculados con las fórmulas. De igual forma, los valores obtenidos mediante la relación $\frac{f}{M}+\frac{A}{L f \times M}$, en todas las condiciones de carga, son mucho mayores a 0,15 , cumpliendo satisfactoriamente con este criterio.

\section{Criterio Holandés}

Para que la embarcación cumpla con esta exigencia, bastará que el brazo adrizante para una escora de $35^{\circ}$ sea igual o mayor de 0,22 m. En la Tabla 8 y Anexo 11 se observa que la condición de carga $\mathrm{C} 7$ es la única que no satisface esta exigencia.

\section{Criterio Japonés}

En la Tabla 9 se resume las exigencias de este criterio frente a los resultados obtenidos para cada una de las condiciones de carga. Las alturas metacéntricas y los francobordos calculados con las fórmulas propuestas, son menores que las correspondientes alturas metacéntricas y francobordos de la embarcación en cada una de las condiciones de carga, cumpliéndose ampliamente con este criterio.

\section{Criterio de Takagui}

En la Tabla 10 se observa que el GM de la embarcación es superior al rango propuesto por el criterio $(0,45 m \leq 0,60$ $m)$, lo cual indica una buena estabilidad. Con respecto a los máximos periodos de balance exigidos por este criterio (Tabla 10) se observa en la, que los periodos de balance de la embarcación son efectivamente menores, cumpliendo bien con esta recomendación. La constante de estabilidad "C" calculada mediante la fórmula (16), se ubica muy a la derecha de la curva de seguridad (Anexos 12), lo cual indica una buena estabilidad. 
Tabla 8. Valores de los brazos adrizantes 1 para las escoras de $35^{\circ}$ frente al mínimo requerido por el Criterio Holandés

\begin{tabular}{cccccccc}
\hline $\begin{array}{c}\text { Requerimiento } \\
\text { del Criterio }\end{array}$ & C1 & C2 & C3 & C4 & C5 & C6 & C7 \\
\hline$G Z_{35^{\circ}} \geq 0,22 m$ & 0,396 & 0,444 & 0,269 & 0,311 & 0,222 & 0,275 & $\mathbf{0 , 1 8 4}$ \\
\hline
\end{tabular}

El valor en negrita no cumple con el mínimo establecido por el criterio.

Tabla 9. Las alturas metacéntricas (GM) y los francobordos (f) calculados mediante las fórmulas propuestas por el Criterio Japonés, frente a las alturas metacéntricas $(G M)$ de la embarcación para todos los estados de carga

\begin{tabular}{|c|c|c|c|c|c|c|c|}
\hline \multirow{2}{*}{ Requerimientos del Criterio } & \multicolumn{7}{|c|}{ Condiciones de carga } \\
\hline & $\mathrm{C} 1$ & $\mathrm{C} 2$ & $\mathrm{C} 3$ & $\mathrm{C} 4$ & $\mathrm{C} 5$ & C6 & $\mathrm{C} 7$ \\
\hline$\overline{\text { GM calculados por la fórmula }}$ & 0,21 & 0,20 & 0,24 & 0,23 & 0,26 & 0,25 & 0,29 \\
\hline$G M \geq M / 23+0,27(\mathrm{~m})$ & 0,447 & 0,446 & 0,449 & 0,448 & 0,449 & 0,449 & 0,449 \\
\hline$G M \geq L f / 120+0,27(\mathrm{~m})$ & 0,349 & 0,348 & 0,352 & 0,352 & 0,353 & 0,352 & 0,353 \\
\hline GM de la embarcación (m) & 0,902 & 0,974 & 0,922 & 0,986 & 0,878 & 0,930 & $\mathbf{0 , 8 0 3}$ \\
\hline$f_{\min } \geq p d / 15+0,2(m)$ & 0,326 & 0,326 & 0,326 & 0,326 & 0,326 & 0,326 & 0,326 \\
\hline f de la embarca & $\mathbf{0 , 8 2 3}$ & $\mathbf{0 , 8 7 0}$ & 0,678 & 0,716 & $\mathbf{0 , 5 2 1}$ & 0,567 & 0,400 \\
\hline
\end{tabular}

Tabla 10. Rangos de la altura metacéntrica $(G M)$, el período de balance $(T)$ y la constante de seguridad " $C$ " propuestos por Takagui frente a los correspondientes valores de la embarcación

\begin{tabular}{cccccccc}
\hline \multirow{2}{*}{ Requerimientos del Criterio } & \multicolumn{7}{c}{ Condiciones de carga } \\
\cline { 2 - 7 } & C1 & C2 & C3 & C4 & C5 & C6 & C7 \\
\hline $0,45 m \leq G M \leq 0,60 m$ & $\mathbf{0 , 9 0 2}$ & $\mathbf{0 , 9 7 4}$ & $\mathbf{0 , 9 2 2}$ & $\mathbf{0 , 9 8 6}$ & $\mathbf{0 , 8 7 8}$ & $\mathbf{0 , 9 3 0}$ & $\mathbf{0 , 8 0 3}$ \\
$T \leq(2 * k t) / \sqrt{ } G M(\mathrm{~s})$ & 4,094 & 3,904 & 4,079 & 3,926 & 4,195 & 4,064 & 4,402 \\
$\boldsymbol{T}(\mathbf{s})$ & $\mathbf{3 , 9 2 4}$ & $\mathbf{3 , 7 8 5}$ & $\mathbf{3 , 8 2 7}$ & $\mathbf{3 , 7 1 9}$ & $\mathbf{3 , 8 5 9}$ & $\mathbf{3 , 7 6 8}$ & $\mathbf{3 , 9 8 2}$ \\
$C=(G M / B G) \times(2 f / M)$ & 0,717 & 0,895 & 0,575 & 0,733 & 0,614 & 0,780 & 0,870 \\
\hline
\end{tabular}

\section{Conclusiones}

La embarcación estudiada, a pesar de su tamaño (eslora menor de $12 \mathrm{~m}$ ) cumple con bastante solvencia la gran mayoría de los Criterios de Estabilidad considerados; notándose que la condición de carga de mayor riesgo es la C7 (Retorno al puerto con la bodega completamente llena y con el $10 \%$ de combustible y agua dulce). En lo referente a las exigencia de la OMI y de Rahola, el brazo adrizante GZ para una escora de $30^{\circ}$ es menor a $0.20 \mathrm{~m}$, solamente alcanza $0,18 \mathrm{~m}$. Lo mismo ocurre con el requerimiento de Criterio Holandés, ya que el brazo adrizante para una escora de $35^{\circ}$ es menor a $0.22 \mathrm{~m}$, solo alcanza $0.18 \mathrm{~m}$. En consecuencia, y dado que cumple con la gran mayoría de los requerimientos de los criterios utilizados, se considera que esta embarcación tiene una buena estabilidad, pudiendo ser mejor si se corrige los problemas que causan la inestabilidad en la condición de carga C7.

\section{Agradecimientos}

Los autores deseamos agradecer al Departamento Académico de Manejo Pesquero y Medio Ambiente de la Facultad de Pesquería de la Universidad Nacional Agraria La Molina por las facilidades brindadas y al Sr. C.
Rumiche, Administrador de la Embarcación, por su apoyo durante la toma de datos, mediciones y la realización de las pruebas de inclinación.

\section{Literatura citada}

Barnaby, K. 1969. "Basic Naval Architecture”. Hutchinson \& CO. (Publishers) LTD. London. 68-85p.

DerretD.R. 1999. "Ship stabilityformasters and mates" Fifth edition. Butterworth Heinemann- Reed Educational and Professional Publishing Ltd. ISBN 0750641010.

Díaz, F.C. 1972. "Teoría del Buque". Editorial Barcelona, España. 219-302p.

FAO/OIT/IMO. 2014. "Recomendaciones de Seguridad para los buques pesqueros con cubierta, de eslora inferior a $12 \mathrm{~m}$ y los buques pesqueros sin cubierta". Roma. Italia. ISBN $N^{\circ}$ 978-92-5307397-9. Disponible en: http://www. fao.org/3/a-i3108s.pdf

FAO, 2018. "El estado mundial de la pesca y la acuicultura". ISBN 978-92-5130688-8 Recuperado de: http://www. fao.org/3/I9540ES/i9540es.pdf

JFBA (Fishing Boats Association of Japan), 1964. In the Journal of the Fishing Boats Association of Japan, $\mathrm{N}^{\circ}$ 130, Tokyo. 66-83p.

Ley General de Pesca (1992), según Decreto Ley $\mathrm{N}^{\circ} 25977$ y su Reglamento aprobado por el Decreto Supremo No 012-2001-PE.

Magallanes, C.A. 2015. "Situación de la Pesca Artesanal en el Perú: Caso de Ica y Piura". Informe Temático $\mathrm{N}^{\circ}$ 132-2014-2015. Congreso de la República.

Mantari, J.; Ribeiro e Silva, S. Guedes S. C. Octubre 2011. "Variation on Transverse Stability of Fishing Vessels due to Fishing Gear Pull and Waves". Centre for Marine Technology and Engineering. Technical University of Lisbon.

Nickum, G.C. (1975). "Proposed Stability Criteria". Fishing Boats of the World (FBW), Vol 1. Four Edition. Fishing News (Boocks). London. 320-32p.

OMI (Organización Marítima Internacional). 2008. "Código Internacional de Estabilidad sin Averías 2008” (IS Code 2008) - Resolución MSC.267(85). Londres.

Rahola, J. 1939. "The judging of the stability of ships and the determination of the minimum amount of stability". Doctoral Thesis. The University of Finland. Helsinki. Yhteiskitjapaino, Osakeyhtio. 232p.

Takagui, A. 1967. “Notes on stability”. Fishing Boats of the World (FBW). 2. Second Edition. Fishing News 
(books). London. p. 475-488.

Womack, J. 2002. "Small Commercial Fishing Vessel Stability Analysis. Where are we Now? Were are we

Going?" Proceedings of the $6^{\text {th }}$ International Ship

Stability Workshop. Webb Institute. New York.

Anexo 1. Las ecuaciones básicas de integración aproximada, sobre cuyas derivaciones se basan los cálculos de las Curvas Hidrostáticas v las Curvas Cruzadas de Estabilidad

\begin{tabular}{lcc}
\hline & Fórmula & Fórmula de Integración Aproximada \\
\hline Área & $A=\int_{-L / 2}^{L / 2} y d x$ & $A=\frac{2 h}{3}\left[y_{0}+4 y_{1}+2 y_{2}+4 y_{3}+\ldots+y_{n-1}+y_{n}\right]$ \\
Volumen & $V=\int_{0}^{H} A d z$ & $V=\frac{H}{3}\left[A_{0}+4 A_{1}+2 A_{3}+\ldots+4 A_{m-1}+A_{m}\right]$ \\
Momento de inercia & $I_{x}=\frac{2}{3} \int_{-L / 2}^{+L / 2} y^{3} d x$ & $I_{x}=\frac{2}{3} \times \frac{H}{3}\left[y^{3}+y^{3}{ }_{2}+y^{3}{ }_{3}+\ldots y^{3}{ }_{n-1}+y^{3}\right]$
\end{tabular}

$\mathrm{H}=$ intervalo entre los niveles de flotación; $\mathrm{m}=$ número de niveles de flotación; $\mathrm{h}=$ intervalos entre las secciones transversales; $\mathrm{n}=$ número de secciones transversales.

Anexo 2. Resultado de los brazos adrizantes supuestos (GsZs) calculados para cada nivel de flotación a escoras entre $0^{\circ}$ y $90^{\circ}$ con intervalos de $10^{\circ}$. Se consideró un $K G s=1,7 \mathrm{~m}$.

\begin{tabular}{cccccc} 
& \multicolumn{5}{c}{ Brazos adrizantes supuestos GsZs (m) } \\
\cline { 2 - 3 } Escoras & Flotación 2 & Flotación 3 & Flotación 4 & Flotación 5 & Flotación 6 \\
& $\mathrm{D}_{2}=7,233 \mathrm{t}$ & $\mathrm{D}_{3}=14,485 \mathrm{t}$ & $\Delta_{4}=23,435 \mathrm{t}$ & $\Delta_{5}=33,689 \mathrm{t}$ & $\Delta_{6}=44,436 \mathrm{t}$ \\
$10^{\circ}$ & 0,1695 & 0,1753 & 0,1701 & 0,1284 & 0,0521 \\
$20^{\circ}$ & 0,3073 & 0,3055 & 0,2769 & 0,2193 & 0,0336 \\
$30^{\circ}$ & 0,3861 & 0,3983 & 0,3378 & 0,1884 & $-0,0023$ \\
$40^{\circ}$ & 0,6237 & 0,4243 & 0,2707 & 0,1370 & $-0,0475$ \\
$50^{\circ}$ & 0,7047 & 0,3525 & 0,1709 & 0,0537 & $-0,0978$ \\
$60^{\circ}$ & 0,6766 & 0,2241 & 0,0534 & $-0,0438$ & $-0,1553$ \\
$70^{\circ}$ & 0,4856 & 0,0770 & $-0,0653$ & $-0,1467$ & $-0,2283$ \\
$80^{\circ}$ & 0,2523 & $-0,0906$ & $-0,1915$ & $-0,2502$ & $-0,3052$ \\
$90^{\circ}$ & $-0,0875$ & $-0,2720$ & $-0,3182$ & $-0,3490$ & $-0,3698$ \\
\hline
\end{tabular}

Anexos 3. Fórmulas utilizadas para el cálculo de los brazos dinámicos utilizando la Regla de 5/8

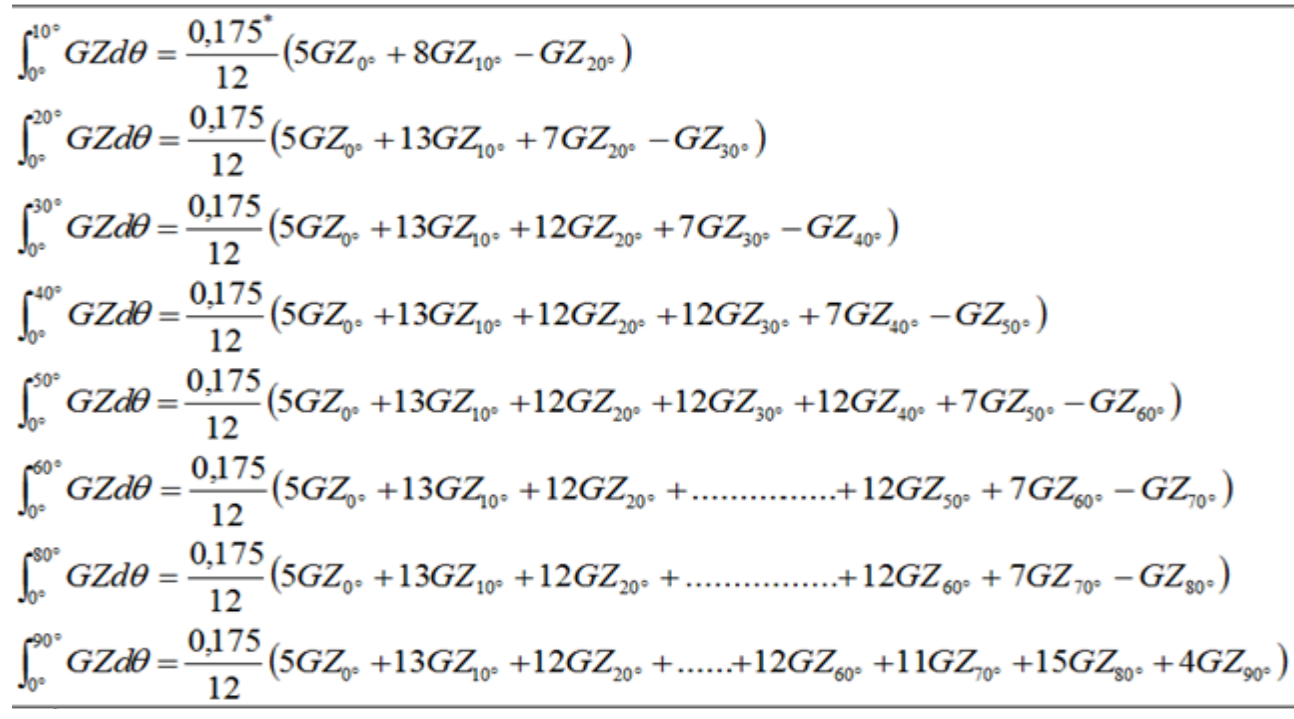

* Intervalo de $10^{\circ}(0,175$ radianes). 
Anexo 4. Valores de los factores $X_{1}, X_{2}$, “ $k$ " $y$ " s" para el cálculo del ángulo de balance " $q_{1}$ ”

\begin{tabular}{|c|c|c|c|c|c|c|c|c|c|c|c|}
\hline \multirow{2}{*}{ Valores factor $\mathrm{X}_{1}$} & $\mathrm{M} / \mathrm{Hm}$ & $\leq 2,4$ & 2,5 & 2,6 & 2,7 & 2,8 & 2,9 & 3,0 & 3,1 & 3,2 & $\geq 3,5$ \\
\hline & $\mathrm{X}_{1}$ & 1,0 & 0,98 & 0,96 & 0,95 & 0,93 & 0,91 & 0,90 & 0,88 & 0,86 & 0,80 \\
\hline \multirow{2}{*}{ Valores factor $\mathrm{X}_{2}$} & $\mathrm{Cb}$ & $\leq 0,45$ & 0,50 & 0,55 & 0,60 & 0,65 & $\geq 0,70$ & & & & \\
\hline & $\mathrm{X}_{2}$ & 0,75 & 0,82 & 0,89 & 0,95 & 0,97 & 1.0 & & & & \\
\hline \multirow{2}{*}{ Valores factor $\mathrm{k}$} & {$\left[\left(A_{K} * 100\right) / L f * M\right]$} & 0 & 1,0 & 1,5 & 2,0 & 2,5 & 3,0 & 3,5 & $\geq 4,0$ & & \\
\hline & $\mathrm{k}$ & 1,0 & 0,98 & 0,95 & 0,88 & 0,79 & 0,74 & 0,72 & 0,70 & & \\
\hline \multirow{2}{*}{ Valores factor $\boldsymbol{s}$} & $\mathrm{T}$ & $\leq 6$ & 7 & 8 & 12 & 14 & 16 & 18 & $\geq 20$ & & \\
\hline & $s$ & 0,100 & 0,098 & 0,093 & 0,065 & 0,053 & 0,044 & 0,038 & 0,035 & & \\
\hline
\end{tabular}

Fuente: OMI, 2008. IS Code 2008. Res. MSC.267(85)

Anexo 5 Secciones transversales de trazos completos (trazos llenos en proa) (líneas punteadas en popa). Escoras de $0^{\circ}$ a $90{ }^{\circ} \mathrm{C}$ con intervalos de $10^{\circ}(0,175$ radianes $)$ F0........F6 =niveles de flotación a intervalos de 0,2917

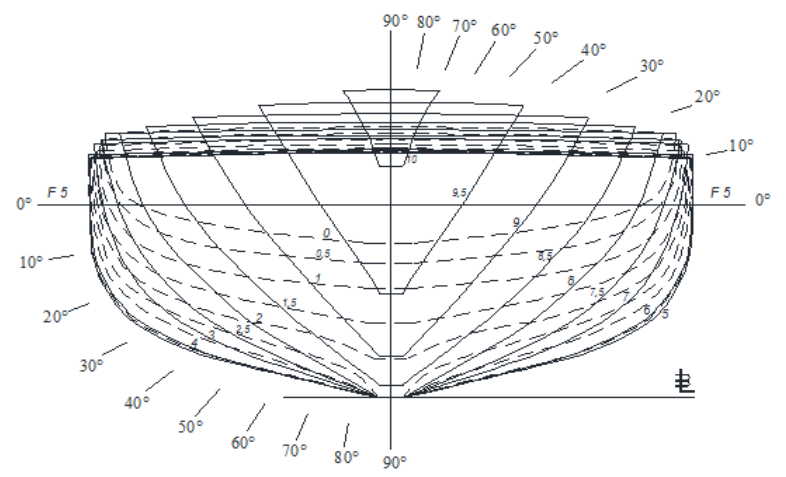

Anexo 6. Esquema que muestra el efecto del viento y el balance intenso en la estabilidad

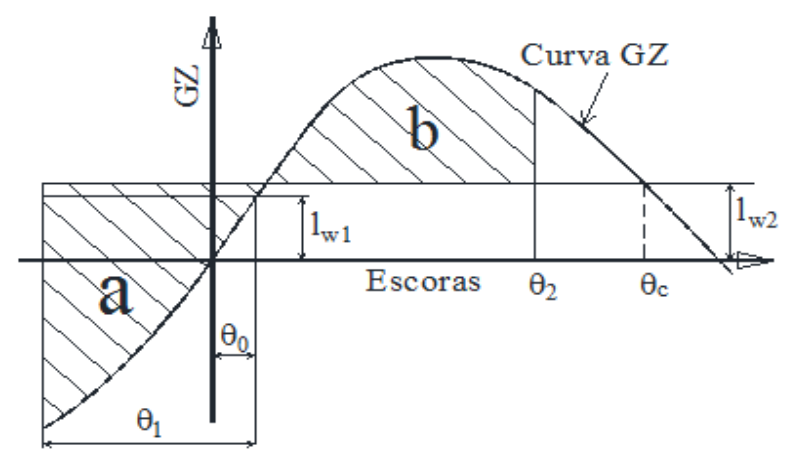

Anexo 7. Curvas de brazos adrizantes para todas las condiciones de carga frente al Criterio de $\mathrm{OMI}(\mathrm{GZ}=0,20$ $\mathrm{m}$ a una escora de $30^{\circ}$ ). C1_RGZ. C2_RGZ, .. C7_RGZ identican a cada una de lascurvas de brazos adrizantes

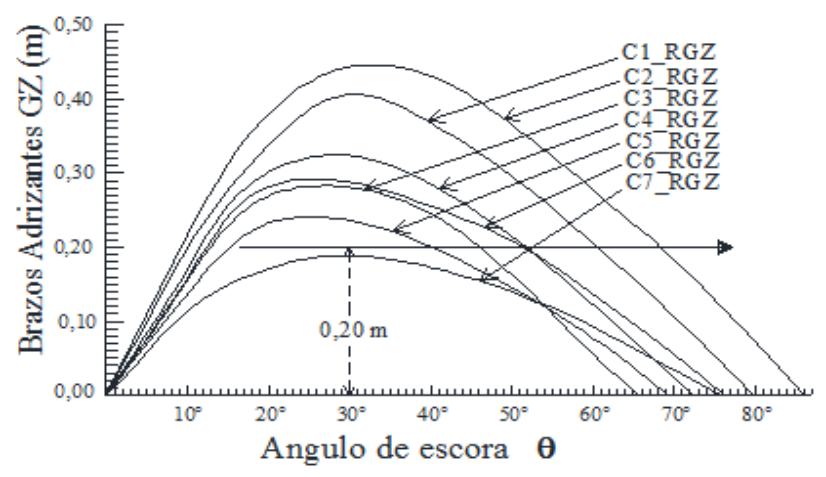

Anexo 8. Curvas de brazos dinámicos para todas las condiciones de carga frente al Criterio de OMI. C1_DGZ, C2_DGZ, C7_DGZ identican a los brazos dinámicos

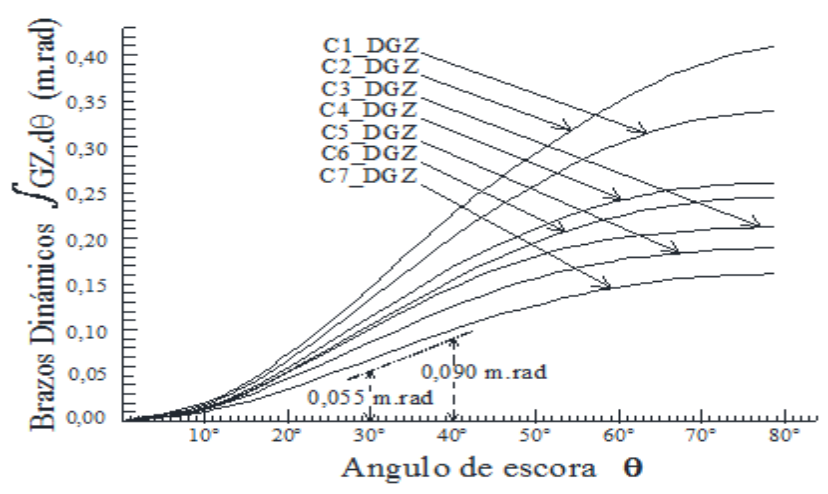


Anexo 9. Aplicación del criterio de Rahola a las curvas de brazos adrizantes para cada una de las siete condiciones de carga

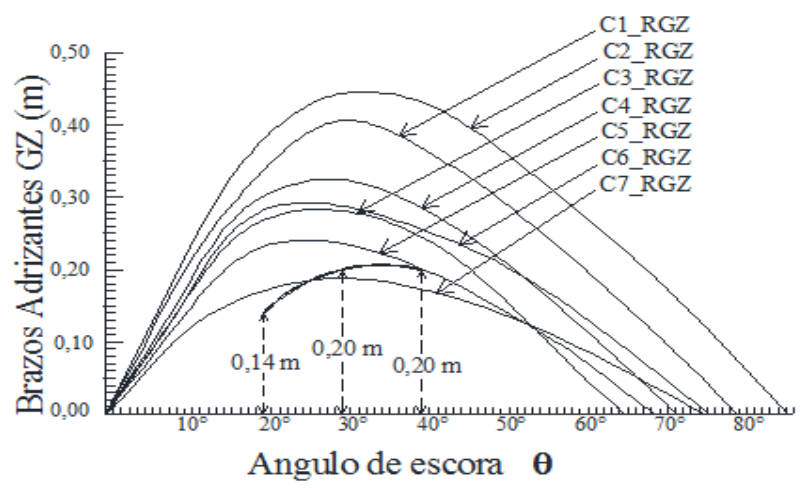

Anexo 10. Aplicación del criterio de Rahola a las curvas de brazos dinámicos para cada una de las siete condiciones de carga

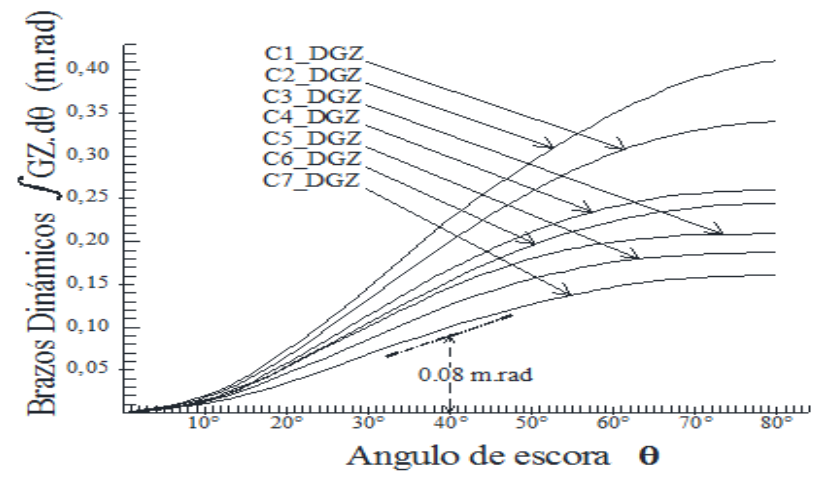

Anexo 11. Criterio Holandés, aplicado a las Curvas de Brazos Adrizantes para las siete condiciones de carga

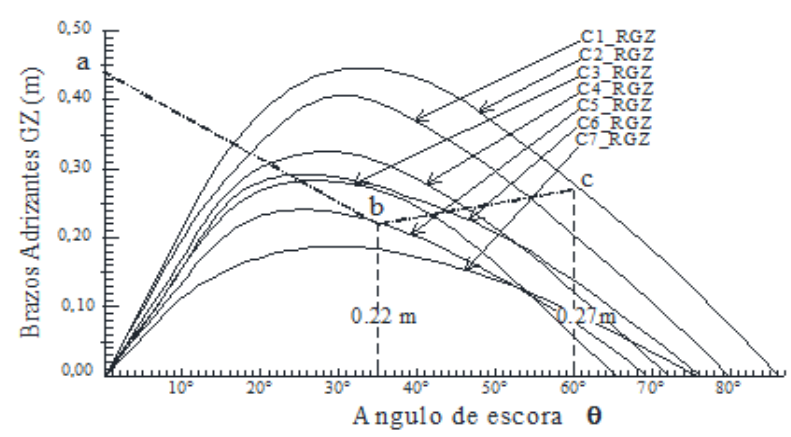


Anexo 12. Criterios de Takagui. El índice de estabilidad "C" ofrece mayor garantía desplazandose hacia la derecha

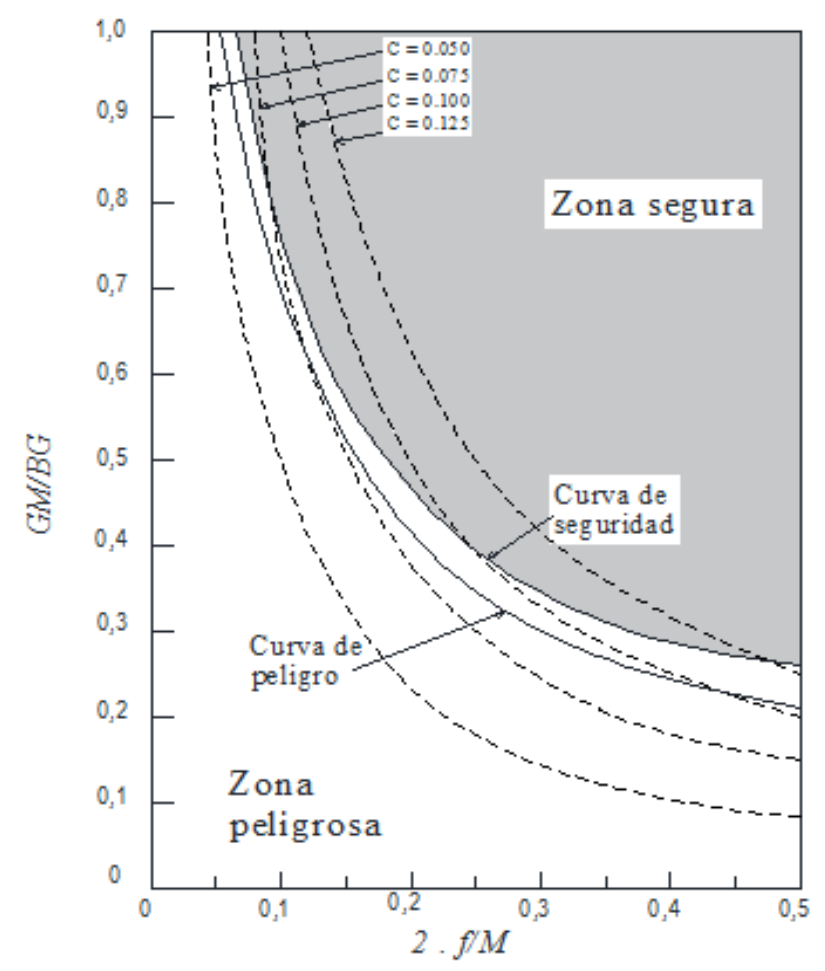

